\title{
Washability of E-Textiles: Failure Modes and Influences on Washing Reliability
}

\author{
Sigrid Rotzler * (D) and Martin Schneider-Ramelow \\ Research Centre for Microperipheric Technologies, Technische Universiät Berlin, Gustav-Meyer-Allee 25, \\ 13355 Berlin, Germany; Martin.Schneider-Ramelow@tu-berlin.de \\ * Correspondence: rotzler@tu-berlin.de; Tel.: +49-30-3147-2889
}

Citation: Rotzler, S.;

Schneider-Ramelow, M. Washability of E-Textiles: Failure Modes and Influences on Washing Reliability. Textiles 2021, 1, 37-54. https:// doi.org/10.3390/textiles1010004

Academic Editor: Philippe Boisse

Received: 7 April 2021

Accepted: 6 May 2021

Published: 21 May 2021

Publisher's Note: MDPI stays neutral with regard to jurisdictional claims in published maps and institutional affiliations.

Copyright: (c) 2021 by the authors. Licensee MDPI, Basel, Switzerland. This article is an open access article distributed under the terms and conditions of the Creative Commons Attribution (CC BY) license (https:/ / creativecommons.org/licenses/by/ $4.0 /)$.

\begin{abstract}
E-textiles, hybrid products that incorporate electronic functionality into textiles, often need to withstand washing procedures to ensure textile typical usability. Yet, the washability-which is essential for many e-textile applications like medical or sports due to hygiene requirements-is often still insufficient. The influence factors for washing damage in textile integrated electronics as well as common weak points are not extensively researched, which makes a targeted approach to improve washability in e-textiles difficult. As a step towards reliably washable e-textiles, this review bundles existing information and findings on the topic: a summary of common failure modes in e-textiles brought about by washing as well as influencing parameters that affect the washability of e-textiles. The findings of this paper can be utilized in the development of e-textile systems with an improved washability.
\end{abstract}

Keywords: electronic textiles; e-textiles; smart textiles; wash testing; washability; reliability; failure modes

\section{Introduction and Context}

If conceived to be worn on or near the body-and even for some non-wearable applications-hybrid electronic textiles (e-textiles), products that incorporate electronic or electrically conductive functionality into textiles, need to be clean- and washable [1] Only e-textiles that exhibit a textile typical usability (which includes washability) will be accepted by users and, thus, lead to commercially successful products [2]. Although many experts agree that a lacking washability is one of the major hurdles that e-textiles need to overcome, there is still only limited research into the topic. While numerous publications on e-textiles do feature wash testing, washability is rarely the main issue, but rather one of a range of reliability tests run to evaluate the developed e-textiles. A difficulty in comparably assessing the washing results that are presented in these publications arises from the very different wash testing methods, an issue that is addressed in a previous publication [3]. While some wash according to, or at least loosely follow, different existing textile washing standards, others use a variety of household washing procedures or even alternative washing methods, like placing the samples in a beaker with water. Not only do the washing methods differ greatly, but also the number of cycles run to assess washability (between one and more than 50 cycles) as well as the use and type of detergent, the washing temperatures, and the subsequent drying of the samples [3].

To improve the washability of e-textile systems, insight is needed into both of the factors influencing the washing outcome as well as the typical damages brought about by washing and weak points in the e-textile that will fail first if subjected to washing procedures. This knowledge will allow researchers and developers a targeted approach towards e-textiles with improved washing reliability. Existing research into those topics is rarely done systematically, as washability is not the main focus in most e-textile related publications, as mentioned above. This review tries to alleviate this lack of insight, by bundling 
findings on influencing factors on washability as well as common failure modes. The presented overview can be used as a basis for improved e-textile designs with enhanced washability. Detailed information regarding the employed washing methods for most of the cited sources can be found in a previous review of wash testing practices for etextiles [3]. An extensive research into relevant scientific publications was used as a basis. Only sources including both wash tests on e-textiles, as well as findings on how different parameters can affect the washability and/or findings on specific failure modes, were included. Similar information on commercially available e-textiles is rarely published; thus, the review is limited to academic research. In a number of publications, e-textiles, which are claimed to be highly washable, are presented-but without any parameter variation. Silver coated cotton by Wand et al. withstands 200 of their washing cycles [4]. Matsouka et al. wash textile electrodes for 50 cycles and retain functionality [5]. The textile based tribo-electric nano-generators in research by Sala de Medeiros et al. show no performance degradation or other damages after 50 washing cycles [6], just to name a few examples. These sources have not been included in the review, because of their widely differing wash testing methods. Because of these different test methods, a comparison between studies - and with it an estimation which factors will lead to an improved washability-is difficult. An e-textile that is claimed to be washable using one test method might not be (as) washable if another testing method is used.

The main review is preceded by a short section on how e-textiles can be negatively affected by washing. Subsequently, different failure modes in e-textiles as a result of washing are presented, with a focus on the specific damages to different structures of an etextile system. The next section lists influencing factors on e-textile washability, including both of the factors originating from the e-textile and its composition as well as the external factors processing and cleaning parameters. A summary of the findings is given at the end of the section.

\section{Effects of Washing on E-Textiles}

During any washing process, the cleanable items are subjected to the four washing factors time, temperature, mechanical action, and chemistry/biology, also known as Sinners factors [7,8]. The magnitude of these interdependent factors determines the outcome of a washing procedure, both the desired effects - cleanliness and hygiene - as well as the unwanted, so-called secondary washing effects (e.g., damages, ageing, or changes in shape or color) [1]. Because of the influence of the four factors in combination with the washing water, an e-textile might exhibit any or all of the following secondary washing effects:

- changes in electrical properties: the loss of conductivity or increase in resistance, altered capacitance [1,9-55]

- changes in function: (partial) loss of function of one or more components (LEDs, ...) or the whole e-textile system $[16,22,40,49,56-62]$

- changes in characteristics: e.g., changes in sensor ranges, antenna spectra, decreased read ranges, $\ldots[10,11,19,30,38,40,52,53,63-72]$

- changes in integrity: e.g., delamination, loosening of wires, loss of components ... $[1,13,20,32,39,42,49,58,61]$

- changes in appearance: e.g., darkening, changes of the textile surface (pilling, fuzzing), wrinkling, shrinkage ... [15,19,22,30,42,53,58,69,72]

The extent of one or more of these changes after a certain number of washing cycles is used as an indicator of washability for e-textiles. The parameter(s) chosen often correlate with the complexity or the composition of the tested system. Most often-especially when testing only conductive textiles-a change in resistance is the parameter of choice to assess washability. Researchers washing antennas or sensors frequently evaluate changes in characteristics and, if added components are present (like LEDs) or fully functional systems are tested, then the estimation of washing reliability is often tied to a change in function [3]. Because maintaining conductivity and/or functionality (within tolerances) is more of an issue when washing e-textiles than the textile integrity, changes in appearance 
and other textile changes are less often employed to assess the washability of e-textiles [3]. Although, in some cases, a change in appearance and changes in resistance go hand-inhand, as in research by Gaubert et al.: the amount of bleaching agents in the detergents used does not only influence the extent of darkening in the examined metallized textiles, but also reduces their conductivity accordingly [19].

\section{Washing Related E-Textile Failure Modes}

When textile integrated electrically conductive or electronic components are subjected to (repeated) washing, damages can occur, which impairs the e-textile's function, appearance, or characteristics. Because e-textiles are a very diverse group of products-not all of which can be found in a literary source featuring wash testing - the following listing is not exhaustive. Likewise, as new e-textiles are developed, previously non-existent failure modes might occur.

\subsection{Damages to Conductive Coatings and Printed Conductive Structures}

Zeagler et al. find that their silver-based printed conductive tracks exhibit cracks after six washing cycles, which results in increased resistance. They reason that the swelling of the hydrophilic cotton substrate, as well as bending occurring during washing, are responsible. In some samples, the printed ink is not only cracked, but also flaking off [55]. Kim et al. wash aramid textiles coated with a graphene and polyurethane (PU) composite. After repeated washing, the graphene is (partially) lost and the fibers of the underlying textile substrate loosened and entangled (see Figure 1a). The collision of the samples with other materials during the washing process is suspected to be the reason [30]. When testing the washing reliability of differently formulated, metal based conductive coatings, Malm et al. observe a loss of and cracks in coating, with the severity of damages-and subsequent increase in resistance-being strongly dependent on the formulation and thickness of the coating (see Figure 1c) [38]. In woven fabric that is coated with graphene based ink, Afroj et al. observe a loss of graphene flakes after washing, which results in an increase in resistance (see Figure 1d) [9]. Shahariar et al. report crack formation of varying severity in their printed-on silver-based paste, as well as a delamination of the paste from the substrates [45]. Other sources also suspect the loss of conductive coating or ink as the reason for increased resistance after washing $[10,48]$. In a larger study with two types of silver-based conductive ink printed onto 14 different woven textiles, Kazani et al. observe a lost ink and resulting increased resistance for all samples, with differing severity dependent on the material combinations (see Figure 1b) [29]. Screen printed antennas in another publication by Kazani et al. also exhibit cracks after washing [67].

\subsection{Damages to Metallization Layers}

Failures that are similar to those described in the previous section occur when washing metallized yarns and textiles. Cai et al. measure the silver content of the washing water of their metallized textile and observe an increase after several cycles, indicating a gradual loss of the silver coating [64]. Dhanawansha et al. also observe lost silver, but find that, with an increasing number of washing cycles, the amount of silver lost decreases, which suggests that silver loss is greatest during the first few washing cycles [65]. In one publication, the loss of metallization is suspected to be the reason for increased resistance after washing, but not further investigated [53]. Rotzler observes a loss of the metallization layer for knitted silver coated nylon fabric, which is dependent on the employed washing program (see Figure 2a) [42]. Similar observations are made by Gerhold [20] and Foerster, who supposes the friction between the test samples and other textiles during washing is responsible for the loss of metallization [18]. Other studies with similar damages to metallized yarns include Tao et al. and Gaubert et al. They observe that the amount of lost metallization increases with the number of washing cycles (see Figure 2b) [49], and it is dependent on the washing parameters (see Figure 2d) [19]. Lee et al. find the amount of metal lost, and related increase in resistance, is dependent on the combination of involved 
materials [34]. uz Zaman et al. presume that mechanical wear is responsible for peeled off and abraded metal layers in metallized nylon fabric [50-52]. When washing gold and copper coated yarns, Schwarz observes cracks in and a loss of metallization, also suspecting mechanical forces as the main cause [43]. Depending on the washing conditions, cracks in the metallization can even occur when the conductive textile is covered by a protective layer of thermoplastic polyurethane (TPU) (see Figure 2c), as a study by Rotzler et al. shows. The authors reason that, due to the conductive textile being covered by a protective layer, mechanical wear and abrasion are not the main cause of damages. Cyclic temperature changes during washing are more damaging to the silver layer, due to a mismatch of the thermal expansion coefficient in the involved materials [1].

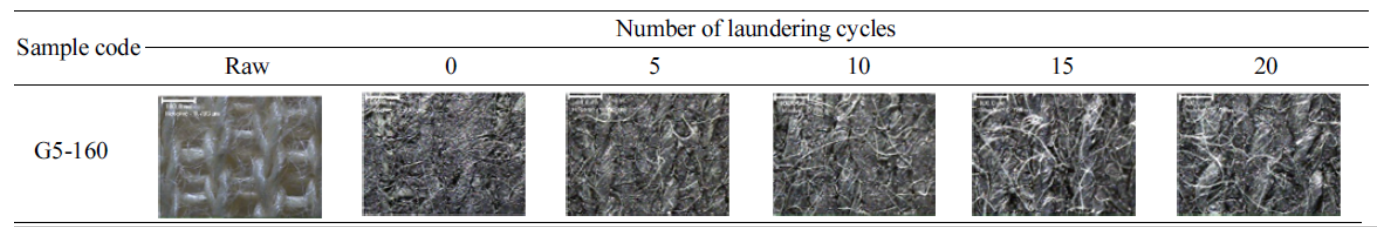

(a) Loss of coating and roughing of textile surface after repeated washing. (Adapted with permission from [30]. Copyright 2018 Springer Nature)

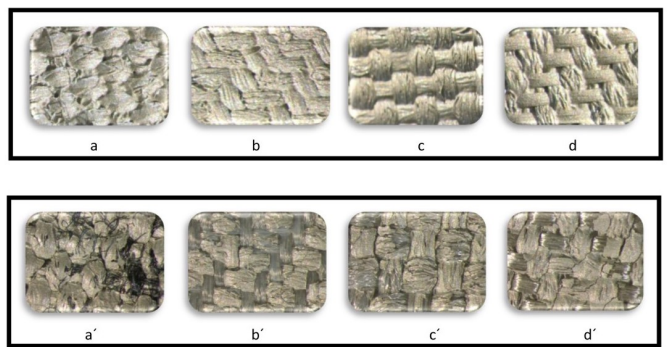

(b) Conductive ink on textiles before (upper row) and after (lower row) washing. (Reprinted with permission from [29]. Copyright 2012 Fibers and Textiles in Eastern Europe)
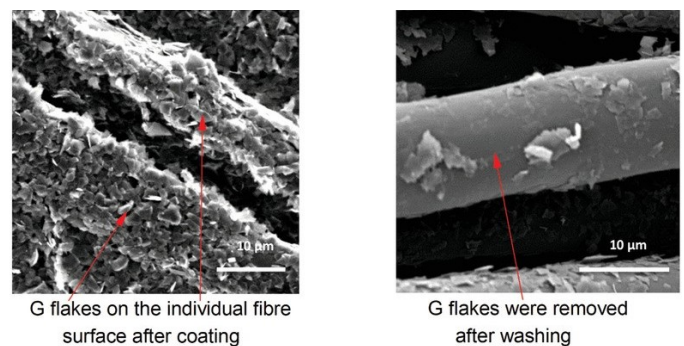
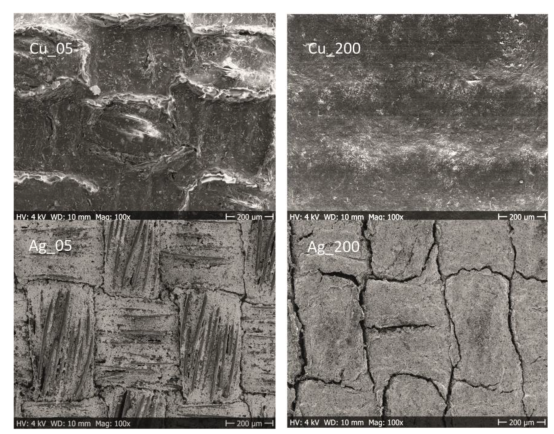

(c) Damage dependent on coating composition and thickness (upper row: copper based, $5 \mu \mathrm{m} \& 200 \mu \mathrm{m}$; lower row: silver based, $5 \mu \mathrm{m}$ \& $200 \mu \mathrm{m})$. (Reprinted from [38])

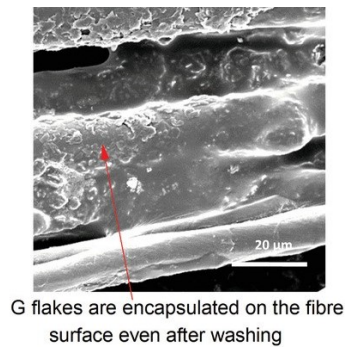

(d) Loss of graphene flakes after laundering, less pronounced with TPU coating. (Adapted from [9])

Figure 1. Examples of damages to conductive textile coatings after washing. 


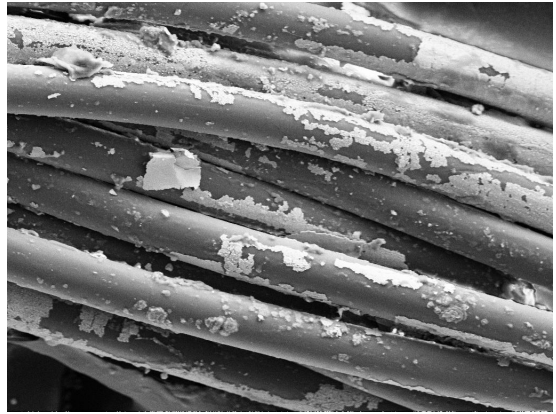

(a) Depleated silver layer on nylon filaments. (Reprinted from[42])

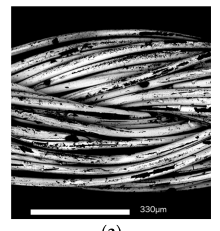

(a)

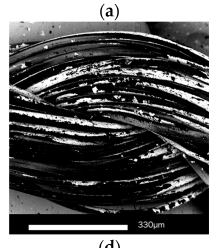

(d)
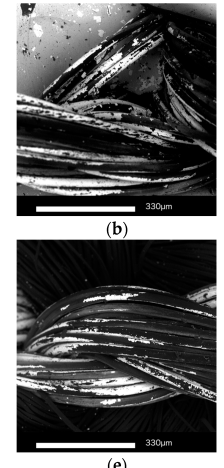

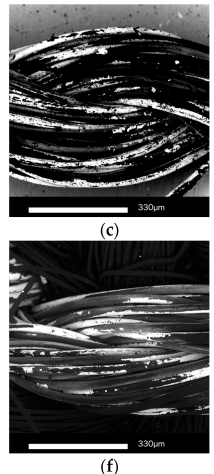

(b) Increased loss of metallization dependent on wash cycle ((a)-(f): before washing, after 5,10,20,40,50 cycles). (Reprinted from [49])

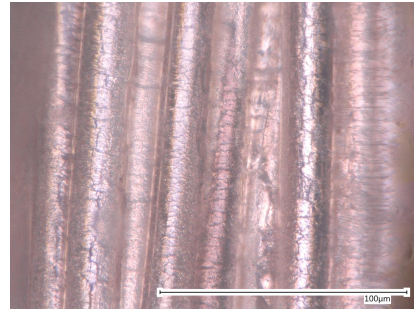

(c) Cracks in metallization layer of TPU encaspulated conductive textiles. (Reprinted from [42])

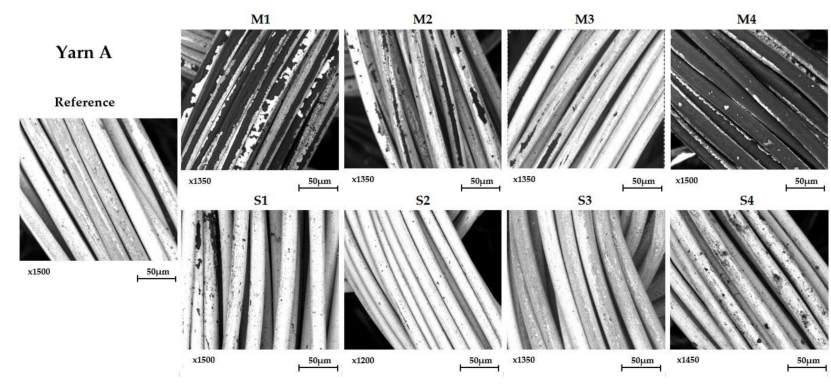

(d) Amount of loss of metallization dependent on washing parameters (1-4: detergent with bleach, detergent without bleach, no detergent, bleaching agent; M: machine wash, S: soaking). (Adapted from [19])

Figure 2. Examples of loss of metallization after washing.

\subsection{Damages to Wires, Conductive Tracks and Connections}

When washing strips of flexible circuit board woven into textiles, Komolafe et al. observe cracks in the copper tracks, located only at the transition zone between tracks and bond pads, identifying these zones as weak points of the circuit [57]. Hardy et al. wash different e-yarns of similar composition-wires that are wrapped around a textile core, with and without added functional components. They see wire breakages along the whole length of yarns, as well as broken and torn off wires at the added components, see Figure 3c [22]. Rotzler et al. conduct a washability study with different types of conductive tracks. In tracks made from meander shaped copper foil embedded in thermoplastic polymer (so-called stretchable circuit boards, SCB), they observe breakages in the copper mostly at the transition from conductive tracks to contacting pads, see Figure $3 \mathrm{~b}$, as well as near the added dummy interposer. Torn litz wires occur in conductive yarn, embroidered onto the textile substrates using the tailored fibre placement (TFP) technique, see Figure 3a. Similar to the results for the SCB tracks, the torn wires occur at a larger number at the contacting points and the interposer, and to a lesser extent along the length of the tracks [1]. SCB tracks that were washed by Veske et al. also exhibit breakages in the tracks, located exclusively at the top and bottom of the meander shapes of the tracks, see Figure 3d [62]. Tao et al. register an increase in broken litz wires corresponding to the number of wash cycles. Another failure mode occurring are broken contacts between the embroidered and interwoven tracks and added LEDs [49].

When washing PU coated copper wires that were knitted into textiles, Li et al. observe breakages of the wires, leading to an increase in the resistance or complete electrical failure. The authors suspect the mechanical strain during washing and resulting deformation of 
the wires to be responsible [35]. Molla et al. find that their embroidered tracks exhibit breakages of the metallized yarn-but the number of breakages is very low when compared to the number of samples with a decreased conductivity, indicating that not only yarn breakages occur, but also a loss of silver coating [39].

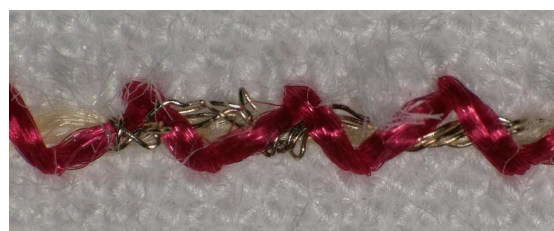

(a) Torn litz wires. (Reprinted from [42])

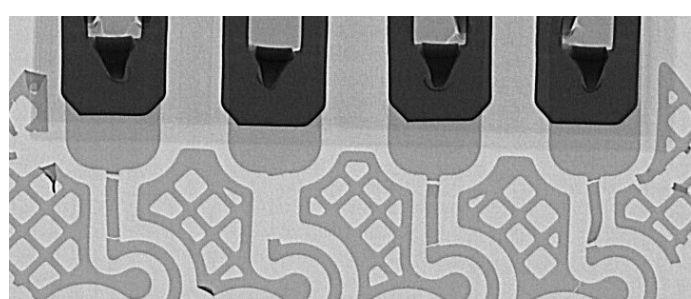

(b) Cracks in copper tracks at the transition to contacting pads. (Reprinted from [42])

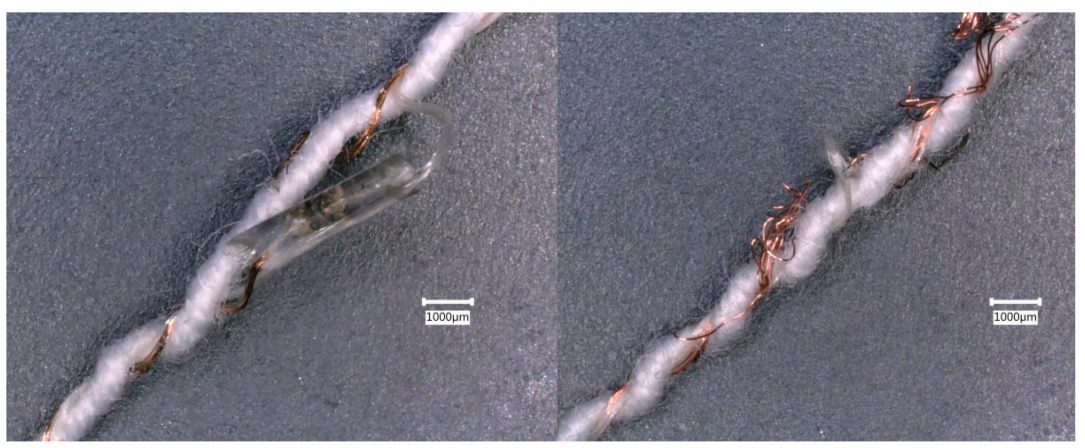

(a)

(b)

(c) Torn litz wires and torn off wires at LED pod. (Reprinted from [22])
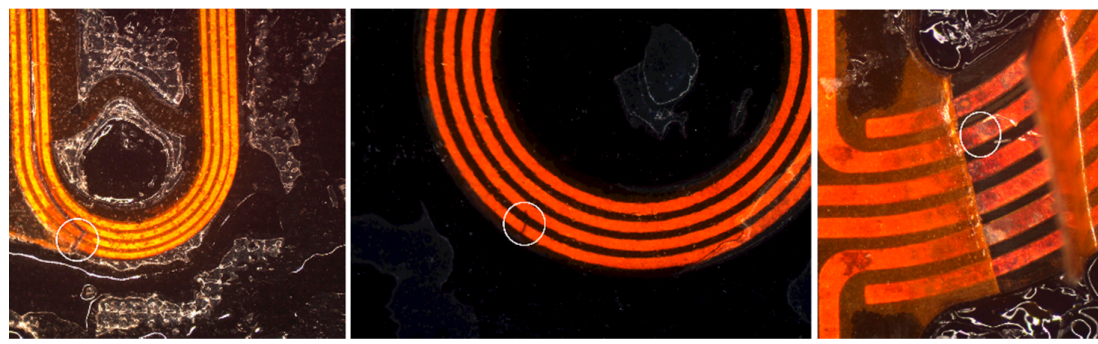

(d) Cracks in copper tracks. (Reprinted from [62])

Figure 3. Examples of damages to wire- and foil based conductive tracks after washing.

Wires embroidered onto a knitted textile using the TFP technique show breakages only at the soldered interconnections to a flexible circuit board in research by Vervust et al. Added LEDs on the board still show function, indicating the board itself and its functional components did survive the washing, only the solder connections are not stable enough [61]. Similar observations are made by Gui et al., their liquid metal tracks are still fully intact after washing, with the only defects presenting at the contacting points between the tracks and added components [56]. Others also report damaged interconnections between tracks and components, circuit boards, or connectors $[1,22,32,39,60]$. In polydimethylsiloxane (PDMS) encapsulated, textile integrated circuits, Ojuroye et al. detect wires that were torn from, as well as chips pulled from, solder joints after repeated washing, being attributed to abrasion, bending, and twisting affecting the samples during the cycles, see Figure 4d [58]. 


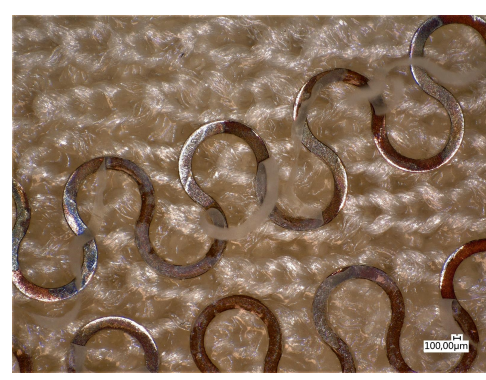

(a) Delamination of protective layer along conductive tracks. (Reprinted from [42])

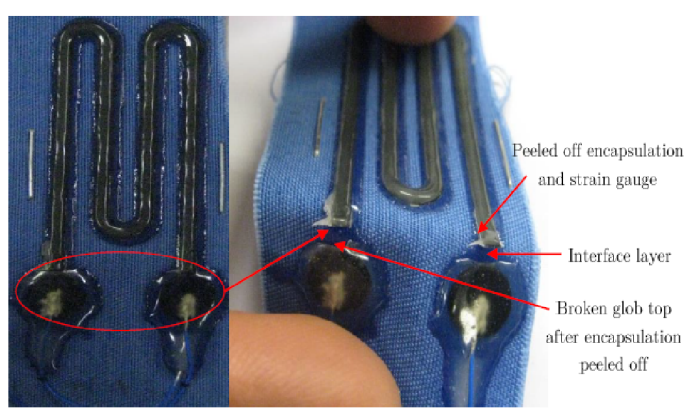

(b) Peeled off glob top protection. (Reprinted with permission from [32]. Copyright 2016 University of Southampton)

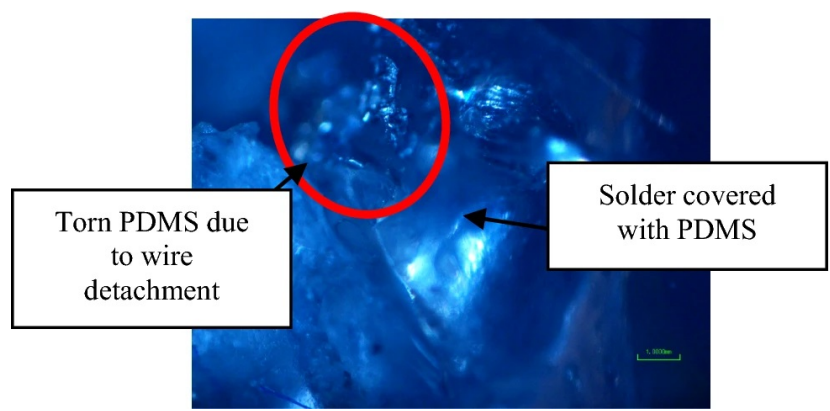

(d) Cracks in PDMS coating. (Reprinted from [58])

(c) Cracks in protective coating. (Reprinted from [71])

Figure 4. Examples of damages to protective layers due to washing.

\subsection{Damages to Protective Layers}

Not only can the conductive materials and components of e-textiles get damaged during washing, but also protective layers or elements. Once these protective structures are corrupted, the underlying conductive or electronic components are more vulnerable to damages themselves [39]. Berglund et al. observe the delamination of the protective PU film in their stitched stretch sensors, with the magnitude of delamination corresponding with an increase in resistance [13]. When washing SCB type conductive tracks, delamination of the protective PU layer occurs along the copper foil tracks in the research conducted by Rotzler. The extent of the damages coincides with the harshness of the washing program, which indicates that frictional forces acting on the samples will lead to thinning and eventual breaks of the PU layer along the edges of the copper tracks (see Figure 4a) [42]. Textile-based radio-frequency identification (RFID) tags in a publication by Wang et al. exhibit a steady decrease in read range when subjected to washing cycles. Cracks in the protective coating of the tags, see Figure $4 c$, which results from mechanical stresses during washing, are suspected to be among the reasons for this reduced functionality [71]. Tao et al. also report the delamination of a TPU layer after washing, leading to an ingress of water and, thus, possibly responsible for increased resistance [49]. When testing different protective films and tapes on embroidered tracks, Molla et al. observe delamination for some of the materials. The exposed tracks and components experience greater damage than intact samples [39]. Komolafe reports peeled-off glob-top and encapsulation layers after washing (see Figure 4b). Another failure mode that is connected to the protective layer occurring in the same publication is the encapsulation peeling off the underlying printed silver layer from an embedded component. The author suggests this is due to a stiffness difference, which makes the contact vulnerable to bending [32]. The textile integrated touch sensors by Ojuroye et al. exhibit an increasingly limited functionality after each washing cycle. A microscopic analysis reveals cracks in the PDMS encapsulation (see Figure 4d), which 
enables water to damage the encapsulated components [58]. Even reversible effects on the protective layers can impact washability. Parkova et al. argue that their silicone coating will stretch during washing, which leads to damages and function loss of embedded LEDs [40], while Gerhold observes the swelling of the PU layer on conductive textiles and suspects that this could lead to decreased conductivity [20].

\subsection{Textile Changes}

When looking at e-textile washability in its entirety, maintaining not only electronic, but also textile, integrity should be considered, even though being less relevant for the continued functionality of the e-textile system. Several sources report changes and damages to the textile substrate. Ojuroje et al. observe wrinkling in their test samples [58], Rotzler pilling [42] - with varying severity depending on washing program and substrate, Quandt et al. entangling of fibers [69], and Martinez-Estrada et al. the loss of fabric pre-treatment after washing [68]. Du et al., Kim et al. (see Figure 1a) and Zhao et al. notice an increase in yarn or textile surface roughing $[15,30,72]$. Xu et al. observe a shrinkage in the wale direction of a knitted metallized textile, which results in a decrease in resistance after one wash cycle [53]. Zhao et al. wash conductive yarn up to 20 times, with shrinkage occurring only during the first two cycles [72], similar findings are made by Hardy et al. [22].

\subsection{Other Failures}

Tao et al. report corrosion on some of their washed conductive yarns, as well as an oxidized battery in an ECG-device [49]. The metallized textiles washed by Gaubert et al. show a darkening of the surface, indicating the oxidation of the silver coating [19]. Rotzler sees a loss of interposers and Ojuroye et al. detached chips [42,58]. Gerhold stops wash testing at the first incidence of detached LED modules from textile based conductive tracks [20].

\subsection{Summary of Failure Modes}

Washing can lead to numerous issues in e-textiles, as this overview of failure modes shows. These failures can occur at specific points in the design or allover, depending on the type and composition of the e-textile. Weak points are contacts between different materials and components-especially if there is a high stiffness gradient-as well as transition areas within the same material (e.g., from conductor track to contact pad). In conductive yarn or textiles without some form of protection, damages can occur throughout the structure. Because added components in the researched publications are mostly encapsulated or otherwise protected, they fail less often than the conductive tracks connecting them or the contacts between tracks and components. For the same reasons, lost or detached components do not occur often. If the protective elements are themselves damaged during washing, though, the components are also vulnerable to damages, e.g., through water ingress. On the other hand, many of the cited sources do not include added components at all, which makes the findings of this paper concerning components less reliable.

\section{Influence of Various Variables on the Washability of E-Textiles}

The following section lists the possible influencing parameters on the washability of e-textiles. These parameters include the specific conductive and non-conductive materials of an e-textile, the methods used to create the e-textile structure, as well as the specific washing and drying procedures employed when testing.

\subsection{Influence of the Textile Substrate}

Ankhili et al. fabricate conductive textiles by coating different non-conductive, knitted textiles with a poly(3,4-ethylenedioxythiophene) polystyrene sulfonate solution (PEDOT:PSS, a conductive polymer). The initial content of conductive material after coating is $6 \%$ for cotton, $4.4 \%$ for nylon, and $3.2 \%$ for the polyester textile. After 50 wash cycles, 
the resistance increases 5-, 7-, and 60-fold, respectively. The authors reason that textile substrates with a higher absorbing capacity for the PEDOT:PSS solution will perform better in subsequent washing tests [10]. Cotton and nylon substrates perform quite similar, though, while resistance for the polyester increases drastically, although the difference of absorbed conductive polymer differs more between nylon and cotton than between nylon and polyester. This indicates that other factors, like the adhesive strength of the coating to the textile material, might also have an influence. When coating three different yarns with PEDOT:PSS, Islam et al. find that pure polypropylene (PP) yarn shows a better washing stability than yarn made from poly(lactic acid) (PLA) or yarn made from a mixture of both PP and PLA [26].

The textile-physical properties (e.g., bending stiffness, thickness, absorbency, ...) of the substrate can impact the washability of textile integrated conductive tracks-according to the specific vulnerabilities of the respective track types. Veske et al. achieve better washability with a thicker textile substrate when testing SCB-type conductive tracks [62]. Rotzler et al. wash three types of conductive tracks on three different textiles: woven cotton, woven polyester, and knitted polyester. Similar to the results shown by Veske et al., SCB type tracks are least washable on the thinnest textile substrate with the lowest bending stiffness (the woven polyester), indicating a vulnerability to bending and folding [1]. Further findings from the same study are that embroidered tracks fare worst on the knitted, stretchable substrate, which suggests a vulnerability to tearing. Laminated-on tracks made from conductive textiles sandwiched between two layers of thermoplastic polymer on the other hand show little to no substrate influence [1]. In contrast, for conductive textiles laminated onto non-conductive textiles using only a bottom layer of TPU, a substrate influence is noticeable: the washability is lowest for the thickest and stiffest textile substrate in research by Lee et al., even though the authors initially suspect the opposite [34]. Because, for this behavior, it could be that the thicker, stiffer denim fabric can be semi-permanently deformed and folded during washing, increasing the abrasion damage at the folds. The much higher elasticity of the other two tested fabrics will lessen fold formation. Molla et al. also find the bending stiffness of the textile substrate and its hydroscopic properties might influence washability for embroidered e-textiles [39].

Komolafe finds that printed circuits show enhanced reliability on anisotropic textiles if printed along the direction of higher stiffness and that the results are worse on elastic substrates [32]. According to Shahariar et al., substrate construction factors into the washing reliability of printed conductive structures. Washability for a non-woven substrate is the lowest, while the woven of two polyethylene terephthalate (PET) textiles outperform the knitted one. They reason that the smaller fiber diameter of the nonwoven fabric reduces ink particle adhesion and, thereby, also the washing reliability [44]. Martinez-Estrada et al. suggest that the loss of the textile finish of their substrate is responsible for the more severe changes in sensor function after the first wash cycle, with subsequent cycles having less impact [68].

\subsection{Influence of Conductive Paste/Ink}

In circuits, sensors, electrodes, or conductor tracks printed onto textiles, the type of metal present, as well as added stabilizers in the ink or paste used, can influence the washability [38]. Adding carbon nano tubes to polypyrrole (an intrinsically conductive polymer) coating increases the washing stability in a study by Frank et al. [17]. Other sources find that the amount and type of non-conductive components in inks and pastes also impact the washing results. A higher PU content, leading to better adhesion, improves the results for Tadesse et al., as does a higher content of a hydrophobic additive [46,47]. Adding TPU and graphene oxide to PEDOT:PSS enhances not only printability, but washability, for Kye et al.; the authors suspect that the elastic TPU matrix hinders penetration of bleaching agents during washing, preventing oxidative damage to the PEDOT:PSS and a subsequent increase in resistance [33]. Hwang et al. improve the washability of silver nano-wire coated silk threads through added PEDOT:PSS. Without the additive, the threads lose all 
conductivity after two washing cycles, in contrast to only doubling the resistance after 10 cycles with added PEDOT:PSS [25]. According to Malm et al., a thicker coating leads to less visual change after washing, and a smoother printed surface will withstand washing better, see Figure 1c [38]. Shahariar et al. suspect that a mismatch of mechanical properties of their printed heating structures and the textile substrates is responsible for unsatisfactory washing performance [45].

\subsection{Influence of Conductive Yarns and Textiles}

Several sources find that the type of the metallization in conductively coated yarns and textiles influences the washing results $[20,31,53,66]$. Gaubert et al. identify yarns with thinner metallization as being less washable [19] and Ankhili et al. suggest that the method of metallizing the yarn-with coating each filament separately prior to twinning leading to better results than coating the finished yarn as a whole-also has an impact on the washability [11]. Similarly, the type of metal used for litz wires in hybrid yarns will influence the washing results according to research conducted by Kivanc et al. [31]. Zeagler et al. show that, for different yarns with the same type of metallization, the one with a higher initial conductivity will withstand washing better [55]. Hardy et al. conclude that the properties of the copper wire used to produce their e-yarns has significant influence on the washability [22].

Apart from the influence of metallization, research from Baribina et al. shows better washability for thicker yarns and worse for textured yarns [12]. Hardy et al. find that yarns with a core of high-tensile vectran fibers are more stable during washing than otherwise identical yarns without [22]. Tao et al. subject differently constructed types of conductive yarns to washing. Litz-wire based yarn shows the worst results, metallized nylon fares slightly better and tinsel-based yarn exhibits the best washability [49]. Parkova et al. obtain similar results when comparing the washability of two metallized yarns and one tinsel-based yarn [40].

\subsection{Influence of Protective Coating}

Many sources look into the benefits of adding protective coating to textile integrated conductive structures. Most of those find that protective coating increases the washing durability of the tested e-textile structures.

The research of Tao et al. shows that a protective layer of TPU on embroidered conductive tracks can slow the rise of resistance that is caused by washing from an exponential increase to a mere linear one [49]. Kazani et al. and Yokus et al. also improve the washability of printed conductive structures by adding a protective TPU layer $[29,54,67]$. The embroidered tracks in Zeagler et al.'s study show better washing performance with an overprinted layer of non-conductive polymer based ink [55]. When testing conductive yarns, Baribina et al. find that a silicone coating yields better washing results than a nano coating, but both are preferable to uncoated yarn [12]. Adding a layer of polyacrylonitrile to textiles that are coated with nano-silver decreases the loss of silver during washing drastically for Dhanawansha et al. [65]. Parkova et al. compare silicone and TPU coating for their embroidered tracks. The results show that the less elastic TPU offers better protection than silicone, as it does not allow the underlying textile and embroidered tracks to stretch as much during washing, but uncoated tracks have the lowest washability [40]. Fu et al. coat RFID antennas that are made from two different types of metallized textiles with fabric glue and moisture protection spray. The spray does not offer any protection from washing damages, while the glue treatment improves washing stability for both of the tested textiles [66]. In a study with five types of encapsulants on embroidered tracks with and without soldered-on LEDs, Molla et al. find significant disparity in the protective effect of the different materials, but all of them lead to a smaller increase in resistance and less defective LEDs when compared to non-encapsulated samples [39]. Linz also achieves different levels of protection for interconnections between the embroidered tracks and PCBs with different encapsulation types [37]. Glob topping offers good washing 
protection for tracks and components on textile integrated flexible circuits according to Komolafe et al. [57]. When washing screen printed tracks, Paul et al. find that glob top encapsulation on the push-button contacts-although it is the less controllable and reproducible method-provides better protection than stencil-printed encapsulation [41]. The results from Malm et al. reveal that a protective coating will have an impact on the washability, but the magnitude of the effect strongly depends on the type of ink that is used to print the underlying conductive structures. For silver-based ink, the coating reduces the loss of conductivity almost five-fold, while the effects for copper based ink are marginal [38]. Afroj et al. find that printing a layer of TPU onto their graphene coated textiles slows the decrease in conductivity after washing-but only in samples that underwent a compression treatment prior to the addition of the TPU [9].

Tao et al. and Veske et al. both achieve better washing results when applying a thicker protective coating [60] or use thicker or double layered TPU foil [62] for their e-textiles. Komolafe finds that, for some applications, increasing encapsulation thickness is not suitable or sufficient for protecting the encased circuits and components. Other strategieslike increasing encapsulation stiffness-have to be employed [32].

Although conductive yarns that were tested by Foerster gain washing stability when adding a layer of TPU after a temperature treatment, the washing results for previously untempered yarn are even better than for those with temperature treatment and TPU protection [18]. In a similar finding, Ankhili et al. conclude that an added TPU layer will not improve the washability for embroidered tracks, due to damages to the conductive yarn that are caused by heat and pressure during TPU application. Overstitching the tracks with a layer of non-conductive yarn offers a much better protection from washing damage for the embroidered tracks [11]. The tested RFID tags that are made from structured metallized textile in Björninen et al.'s research fare worse with added acrylic coating - but the authors suggest that a more suitable encapsulation material might lead to an improvement of washability [63].

\subsection{Influence of Layout, Design \& Integration}

Erdem et al. find that longer transmission lines lead to decreased washing reliability-the resistance in longer lines increases at a faster rate than in shorter ones [16]. Printed conductor tracks on textiles will have improved washability if the line width is increased, as research by Huang et al. shows [24]. Veske et al. test SCB type conductive tracks with different meander shapes for their washability. The study reveals notable differences for the different shapes and the protective quality of adding nonfunctional, re-enforcing structures, see Figure 5a [62]. Packaged pairs of LEDs exhibit a better washability in illuminated e-yarns from Hardy et al. than LEDs added onto flexible circuit strips. In general, their research shows that the size of integrated components in different types of e-yarns correlates with the washability: the smaller the components, the better the washability [22].

Research from Linz, uz Zaman et al. as well as Zeagler et al. shows that, in embroidered conductive tracks, multiple embroidered lines on top of one another improve the washabililty as compared to a single embroidered line [37,51,55]. For anisotropic textile substrates, the orientation of the lines (warp, weft, or diagonal) influences the washing outcomes for sewed or embroidered tracks [27]. Zeagler et al. also find that an additional printed layer of conductive ink improves washability for embroidered tracks. Printing over the embroidered line yields better results than a printed layer under the line [55]. The washing results for embroidered tracks in research conducted by Molla et al. are worse for tracks with added LEDs when compared to tracks without added components [39]. When contacting printed circuit boards (PCBs) with embroidered yarn, Linz finds that pre-drilling holes in the contact pads of the PCBs yields less reliable contacts after washing than piercing the pads. Using adhesive bonding technology to connect the embroidered tracks to the PCB leads to even better results [37]. 

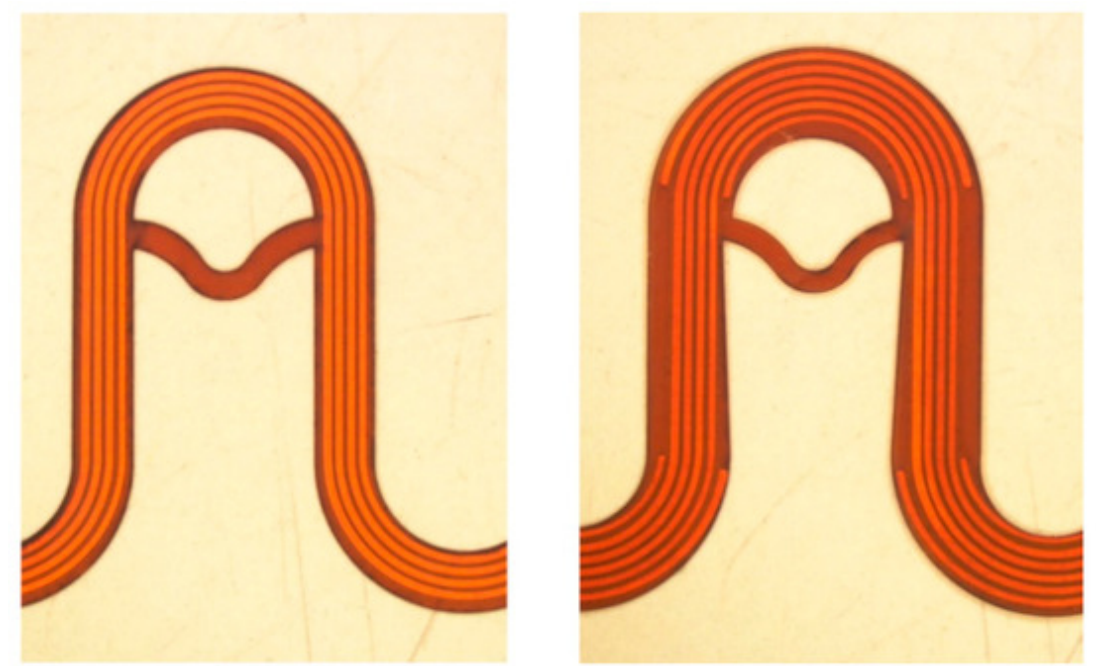

(a) Non-functional copper reinforcement structures (right image) significantly increase washability. (Adapted from [62])
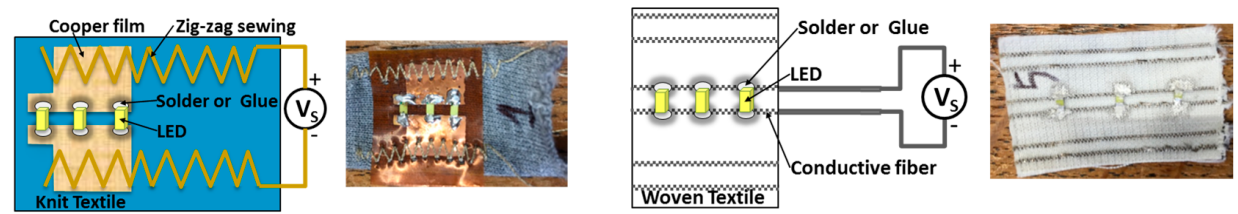

(b) Different system designs for textile integrated LEDs. (Adapted from [49])

Figure 5. Impact of layout and integration method.

Hardy et al. wash acoustic e-yarns that are integrated either into woven tubes or into sewn pockets of their textile substrates and conclude that the woven tubes offer better washing stability [22]. Similarly, Parkova et al. obtain different washing results that are dependent on the integration method: when testing conductive yarns either woven or sewn into textiles, the light intensity of LEDs soldered onto the tracks decreases slower after consecutive washing cycles for the woven tracks compared to the sewn tracks [40]. Tao et al. add LEDs to both sewn and woven tracks, using two different contacting methods (solder and glue), with and without silicone encapsulation. The LEDs on the woven tracks are directly contacted to the integrated conductive threads, while, for the sewn tracks, a flexible PCB with copper foil contact pads is used, see Figure 5b. Here, the systems on woven tracks, especially in combination with soldered joints, are less washable than the ones on the sewn tracks: after 18 wash cycles, five of 18 LEDs lost function, while all of the LEDs on sewn tracks still work. The authors argue that the PCB provides stability, thus making the system more washable. In addition, they suspect that the soldered joints for the woven tracks are less washable, because the nickel plating of the integrated wires makes the solder brittle [49]. Washability decreases if conductive yarns that are added to knitted fabric are spaced further apart from each other in a study conducted by Kayacan et al. [28].

\subsection{Influence of Processing Parameters}

Kim et al. coat aramid knit with a graphene based solution. The temperature of a subsequent hot press treatment influences not only the initial resistance of the textiles, but also the amount of conductive coating lost after repeated washing. The non-linear effect leads to the best results for a medium warm press [30]. Afroj et al. increase the washability of their graphene coated e-textile by subjecting them to a compression treatment after coating. The effect of this treatment leads to a better washability than an additional TPU coating, with a combination of both measures achieving the greatest improvement [9]. The copper coated PET textiles in research by Liu et al. show a much better washability when coated by electroless deposition as compared to thermal evaporation coating [36]. 
When pre-treated with different temperatures, the washing reliability for conductive threads changes in a study conducted by Foerster. The higher the temperature, the worse the results [18]. Rotzler finds that temperature and pressure acting on metallized textiles during a lamination process to attach them to a textile substrate, damaging the metallization layer. This damage subsequently leads to reduced washability [42]. The lamination parameters can also affect washing reliability in other ways: if lamination temperature and duration for polymer-embedded SCBs are not high enough, then the adhesion to the textile is not sufficient and tracks laminated onto the textile substrate can detach during washing. On the other hand, if the temperature is too high, the polymer embedding softens too much during the lamination process, which results in an excess permeation of polymer into the textile substrate-especially with textile substrates having larger pores, like knitted textiles. This leaves the tracks vulnerable to washing forces due to a reduced thickness of the protective polymer layer (see Figure 4a) [42]. Hirman et al. connect surface mounted resistor chips to textile integrated tracks. The washability improves slightly when the textile is stretched prior to contacting, while soaking the contacting point with glue has no discernible effect [23].

\subsection{Influence of Washing \& Drying Parameters}

Washing reliability is not only influenced by parameters pertaining to the construction and processing of e-textiles themselves, but also the employed washing and drying methods. The results of Hardy et al. and Berglund et al. show that tumble drying leads to more damages than washing $[13,22]$. Three separate studies find that placing the e-textiles inside a washing bag increases the washability [35,61,72].

$\mathrm{Xu}$ et al. wash different metallized textiles with three types of detergents and one fabric softener. For the woven e-textile, surface resistivity increases, depending on the additive, while little change is observed for the knitted textile [53]. Gaubert et al. also test knitted metallized textiles. When washing them with different detergents, the results reveal that the specific detergent ingredients can strongly influence the washing reliability: the presence of bleach not only leads to sample darkening due to oxidation, but also to a significantly larger increase in resistance when compared to the detergent without bleaching components. In addition to machine washing, the samples are also put into wash liquor that is mixed from water and the different detergents. This immersion takes place at room temperature without agitation. These comparative tests reveal that, when only two of Sinner's factors (chemistry and time) affect the samples at a time, the change in resistance (and appearance) is minimal even with bleaching agents present. Figure $2 \mathrm{~d}$ shows that the amount of lost metallization strongly depends on the washing parameters [19]. Guo et al. wash PEDOT:PSS wires printed onto nonwoven fabric in deinoized water with and without added soap. Half of the samples are sprayed with a hydrophobic treatment prior to washing. Adding soap increases the resistance more than $100 \%$ after three cycles as compared to only $25 \%$ for water only. With the hydrophobic treatment, the resistance increase is minimal, even with soap [21]. Although an improvement in washability is achieved, the use of hydrophobic agents is questionable, as it will be possible to also inhibit soil release, which makes the treated e-textiles hard to clean.

After putting their printed circuits in water with detergent or heated water without detergent for several hours, the samples from Gui et al. still maintain functionality. After one cycle in the washing machine (with and without detergent), the samples fail [56]. Uz Zaman et al. observe that differently metallized textiles show different reliability when put in water with and without detergent. While silver-plated textiles are almost unaffected by the treatment, resistance for textiles with copper-based metallization increases for both water and water with added detergent [52]. For Lee et al., the resistance of four conductive textiles with different types of metallization remains stable when soaking them in water with added detergent for several days. Machine washing on the other hand leads to an increase in resistance dependent on the metallization and the substrate [34]. The performance of printed textile RFID tags decreases faster after soaking in wash liqour at $40{ }^{\circ} \mathrm{C}$ as 
compared to being placed in just water at $60^{\circ} \mathrm{C}$ in research by Virkki et al. [70]. Similarly, Choi et al. find that the resistance of their PEDOT:PSS coated thread quadruples after four cycles in a detergent solution, while the increase is minimal after the same treatment with only deinoized water [14].

Ojuroye et al. wash their flexible circuits that were woven into a textile substrate with several washing programs, resulting in different outcomes. They suspect that a higher spin speed is responsible for an increased washing damage [58]. Because the compared programs also differ in other aspects, it is unclear whether the spin speed (alone) leads to this reduced washability. Li et al. also find a gentle washing program to be more suitable for the washing of conductive wires knitted into textiles [35], as do uz Zaman et al. when testing the washability of textile electrodes [52]. Another study by the same authors also produces better results for embroidered conductive tracks with a (gentle) silk washing program compared to a harsher express washing program. The outcome depends on the conductive yarn, though, as one of the tested yarns shows no increase in resistance for either washing program [51]. Textile solar cells in the research by Satharasinghe et al. exhibit equal washability when subjected to either hand or machine washing [59]. Frank et al. find that an increased temperature or washing duration as well as the composition of the detergent (worse washability with added bleach — whiin accordance with the results of Gaubert et al.) will lead to more washing damages [17]. An extended study on the washability of textile integrated conductor tracks by Rotzler et al. finds mechanical action during washing to be the overall most damaging of the four Sinner's factors, although the results depend strongly on the type of circuit path. In contrast to SCB and embroidered tracks, for circuit paths made from polymer embedded conductive textile, a high washing temperature leads to a worse washing result than high mechanical action. This is attributed to a mismatch of the coefficient of thermal expansion between the textile filaments, the metallization and the polymer layers [1].

\subsection{Summary of Washability Influences}

The textile substrate's properties will in many cases impact washing reliability of e-textiles. Which of the properties are the most influential is dependent on the type of e-textile. For printed and coated structures, the absorbency, adhesion behavior, as well as textile surface and structure need to be considered. For embroidered or laminatedon conductors, the physical and mechanical properties-(structural) elasticity, bending stiffness, and thickness - of the textile base have to match the requirements that are brought about by specific vulnerabilities of the type of conductor used. Alternatively, in reverse conclusion, if the specific application demands a textile substrate with certain properties (e.g., sufficient stretchability for sports clothing), then the type of conductor shou ld be chosen accordingly (e.g., in the case of elastic substrate, it should not be prone to tearing).

An improved washability for conductive metal-based and polymer-based coatings will be achieved through enhanced adhesion to the textile substrate, enhanced cohesion withing the coating as well as a not to thin, homogeneous coat.

The structure of an e-yarn will influence its washability. Conductive elements should be as robust as possible and processed in a way that allows for maximum contact between multiple conductive litz wires, coated filaments, tinsel, etc. For similarly designed yarns, those with lower resistance (achieved through additional conductive material) will withstand washing better. The results also suggest that a smoother yarn surface will lead to a better washing reliability. In addition, non-conductive structures that add mechanical stability, like high-tensile core fibers or additional fibers for increased yarn diameter and a subsequent increase of the minimal bending radius, will improve the washability.

In most of the reviewed sources, the addition of protective elements leads to improved washability. The results suggest, though, that care has to be taken not to damage the underlying conductive structures when applying the protection. The protective coatings or structures do not only act as a barrier for the conductive elements, preventing abrasion or loss of material. They can also provide additional mechanical stability, which 
makes the overall e-textile more robust. Therefore, the properties of the protective coatings (e.g., bending stiffness, thickness, and elasticity) have to be coordinated with the type of e-textile and textile substrate used. The results suggest that, even when placed under the conductive elements, protective structures can improve the washability not by acting as an encapsulation, but by locally stiffening and strengthening the system, leading to improved mechanical robustness. Because most protective elements will (locally) add bulk and weight and decrease the breathability and flexibility [61], a balance needs to be found between maximum protection for the integrated conductive and electronic structures and maintaining textile characteristics and comfort.

In line with the findings concerning yarns and coatings, conductive elements of etextiles withstand washing better if they are thicker, doubled, and/or reinforced with supporting structures. The more complex the system, the more challenging the washability. Components and interconnections should be designed as small and short as possible, and the amount of components added should be minimal. The integration method needs to be suitable for the chosen conductors and components, and it needs to ensure sufficient mechanical stability.

Processing parameters need to be carefully coordinated with the specific type of conductive or electronic materials. Finding the correct parameters can be difficult, as e.g., too little pressure, temperature, time, etc. can lead to insufficient adhesion or fixation, while too much can result in damages.

The washability of e-textiles is strongly dependent on the washing (and drying) procedure chosen to evaluate the washability. Increased Sinner's factors (time, temperature, mechanical action, and chemistry/biology) lead to increased washing damage. If testing does not expose the e-textiles to all four of the factors at the same time, the results will not necessarily be transferable to regular machine washing, a process involving all four factors in varying magnitude. Thus, a simplified test might lead to biased estimates of washing reliability. Based on the gathered results, a gentle washing program will lead to better washability for (most) e-textiles. When choosing a wash testing program, care has to be taken, though, to ensure that the chosen program fits the e-textile and its application. If the program is too gentle to properly clean application-specific stains off the e-textile, then it is not suitable to evaluate the washability [1,3]. Although mechanical action has been identified as being the overall most damaging washing factor (also see Section 3), it is advisable to evaluate which factor to increase or decrease dependent on the type of e-textile. The results of the reviewed publications reveal that the same washing procedures can impact different e-textiles differently.

\section{Conclusions}

This article provides an extensive overview of common damage types in e-textiles as a result of (repeated) washing, as well as a collection of influence factors on e-textile washability. Although not without limitations, the collective findings provide much needed insight into how washing affects e-textiles. In doing so, this article can serve as a basis to tackle the pressing matter of washability from different angles and, thereby, create more reliable e-textiles. While some of the findings are applicable to most e-textiles, others are only valid for a specific type of textile-integrated electronics. For a future in-depth approach towards reliably washable e-textiles, a more differentiated examination will thus be necessary, discerning conductor and substrate materials and their requirements as well as the specific e-textile application more thoroughly than this relatively general overview. To achieve this, it will not be sufficient to access, in some cases, the very limited existing sources-as was done in this review. Instead specific, targeted research into single aspects of e-textile washability needs to be conducted. Protective elements that do not impair the textile properties-maybe even made from textile material themselves, integrating miniaturized electronic components on a fiber or yarn level as well as extensive wash testing with a variation of the Sinner's factors are some of the solutions that seem promising, based on the results that are presented in this review. 
Author Contributions: Conceptualization, methodology, investigation, resources, writing-original draft preparation, S.R.; writing-review and editing, S.R. \& M.S.-R.; visualization, S.R.; supervision, M.S.-R. All authors have read and agreed to the published version of the manuscript.

Funding: This research received no external funding.

Institutional Review Board Statement: Not applicable.

Informed Consent Statement: Not applicable.

Data Availability Statement: No new data were created or analyzed in this study. Data sharing is not applicable to this article.

Conflicts of Interest: The authors declare no conflict of interest.

\section{References}

1. Rotzler, S.; Kallmayer, C.; Dils, C.; von Krshiwoblozki, M.; Bauer, U.; Schneider-Ramelow, M. Improving the washability of smart textiles: Influence of different washing conditions on textile integrated conductor tracks. J. Text. Inst. 2020, 111, 1766-1777. [CrossRef]

2. Yole Développment. Wearables in Consumer and Medical Applications 2020; Techreport; Yole Développment: Lyon, France, 2020.

3. Rotzler, S.; von Krshiwoblozki, M.; Schneider-Ramelow, M. Washability of e-textiles: Current testing practices and the need for standardization. Text. Res. J. 2021. [CrossRef]

4. Wang, L.; He, D.; Li, J.; He, B.; Qian, L. Conductive cotton fabrics with ultrahigh washability by electroless silver plating after silane modification. Cellulose 2021. [CrossRef]

5. Matsouka, D.; Tao, X.; Koncar, V.; Bahadir, S.K.; Kalaoğlu, F.; Jevšnik, S. Electrical connection issues on wearable electronics. In Proceedings of the Aegean International Textile and Advanced Engineering Conference (AITAE2018), Mytilene, Greece, 5-7 September 2018; IOP Publishing: Bristol, UK, 2018; Volume 459, p. 012017. [CrossRef]

6. Sala de Medeiros, M.; Chanci, D.; Moreno, C.; Goswami, D.; Martinez, R.V. Waterproof, Breathable, and Antibacterial Self-Powered e-Textiles Based on Omniphobic Triboelectric Nanogenerators. Adv. Funct. Mater. 2019, 1904350. [CrossRef]

7. Wagner, G. Waschmittel, 5th ed.; Wiley VCH: Weinheim, Germany, 2017.

8. Ellmer, K. Wäsche-Cluster in Konsumentenhaushalten. Ph.D. Thesis, Technische Universität Berlin, Berlin, Germany, 2016.

9. Afroj, S.; Tan, S.; Abdelkader, A.M.; Novoselov, K.S.; Karim, N. Highly Conductive, Scalable, and Machine Washable GrapheneBased E-Textiles for Multifunctional Wearable Electronic Applications. Adv. Funct. Mater. 2020, 2000293. [CrossRef]

10. Ankhili, A.; Tao, X.; Cochrane, C.; Coulon, D.; Koncar, V. Washable and Reliable Textile Electrodes Embedded into Underwear Fabric for Electrocardiography (ECG) Monitoring. Materials 2018, 11, 256. [CrossRef]

11. Ankhili, A.; uz Zaman, S.; Tao, X.; Cochrane, C.; Koncar, V.; Coulon, D. Washable embroidered textile electrodes for long-term electrocardiography monitoring. Text. Leather Rev. 2019, 2, 126-135. [CrossRef]

12. Baribina, N.; Baltina, I.; Oks, A. Application of additional coating for conductive yarns protection against washing. Key Eng. Mater. 2018, 762, 396-401. [CrossRef]

13. Berglund, M.E.; Coughlin, J.; Gioberto, G.; Dunne, L.E. Washability of E-Textile Stretch Sensors and Sensor Insulation. In ISWC '14: Proceedings of the 2014 ACM International Symposium on Wearable Computers; Association for Computing Machinery: New York, NY, USA, 2014; pp. 127-128. [CrossRef]

14. Choi, C.M.; Kwon, S.N.; Na, S.I. Conductive PEDOT:PSS-coated poly-paraphenylene terephthalamide thread for highly durable electronic textiles. J. Ind. Eng. Chem. 2017, 50, 155-161. [CrossRef]

15. Du, D.; Tang, Z.; Ouyang, J. Highly washable e-textile prepared by ultrasonic nanosoldering of carbon nanotubes onto polymer fibers. J. Mater. Chem. C 2018, 6, 883-889. [CrossRef]

16. Erdem, D.; Yeşilpınar, S.; Şenol, Y. Design of a TENS knee pad with integrated textile electrodes. In Proceedings of the 7th International Technical Textiles Congress, Izmir, Turkey, 10-12 October 2018; pp. 237-245.

17. Frank, E.; Bauch, V. Hochfunktionale leitfähige Beschichtungen aus CNT/Polypyrrol-Kompositbeschichtungen für Intelligente Textilien; Resreport; Institut für Textilchemie und Chemiefasern, Deutsche Institute für Textil- und Faserforschung: Denkendorf, Germany, 2013.

18. Foerster, P. Untersuchungen zu Eigenschaften von Nanosilberschichten auf Polyamidfasern; Studienarbeit, Technische Universität Berlin: Berlin, Germany, 2010.

19. Gaubert, V.; Gidik, H.; Bodart, N.; Koncar, V. Investigating the Impact of Washing Cycles on Silver-Plated Textile Electrodes: A Complete Study. Sensors 2020, 20, 1739. [CrossRef] [PubMed]

20. Gerhold, L. Researches on Ultrasonic-Welded Electrical Interconnections at Smart Textiles Using Nonconductive Adhesives. Master's Thesis, Hochschule für Technik und Wirtschaft HTW Berlin, Berlin, Germany, 2018.

21. Guo, Y.; Otley, M.T.; Li, M.; Zhang, X.; Sinha, S.; Triech, G.M.; Sotzing, G.A. PEDOT:PSS 'Wires' Printed on Textile for Wearable Electronics. ACS Appl. Mater. Interfaces 2016. [CrossRef]

22. Hardy, D.A.; Rahemtulla, Z.; Satharasinghe, A.; Shahidi, A.; Oliveira, C.; Anastasopoulos, I.; Nashed, M.N.; Kgatuke, M.; Komolafe, A.; Torah, R.; et al. Wash Testing of Electronic Yarn. Materials 2020, 13, 1228. [CrossRef] 
23. Hirman, M.; Navratil, J.; Steiner, F.; Hamacek, A. Effect of Washing Cycles on Glued Conductive Joints Used on Stretchable Smart Textile Ribbons. In Proceedings of the 2020 IEEE 8th Electronics System-Integration Technology Conference (ESTC), Tonsberg, Norway, 15-18 September 2020; pp. 1-4. [CrossRef]

24. Huang, T.H.; Tao, X.; Ko, Y.C.; Hsu, P.C.; Shen, C.L.; Chen, F.L.; Wang, W.C.; Jou, G.T.; Koncar, V. A Novel Design of E-Textile Integration for Physiological Monitoring and Lighting. J. Fash. Technol. Text. Eng. 2018, S4, 10. [CrossRef]

25. Hwang, B.; Lund, A.; Tian, Y.; Darabi, S.; Müller, C. Machine-Washable Conductive Silk Yarns with a Composite Coating of Ag Nanowires and PEDOT:PSS. ACS Appl. Mater. Interfaces 2020, 12, 27537-27544. [CrossRef]

26. Islam, G.M.N.; Collie, S.; Qasim, M.; Ali, M.A. Highly Stretchable and Flexible Melt Spun Thermoplastic Conductive Yarns for Smart Textiles. Nanomaterials 2020, 10, 2324. [CrossRef]

27. Ismar, E.; uz Zaman, S.; Bahadir, S.K.; Kalaoglu, F.; Koncar, V. Seam Strength and Washability of Silver Coated Polyamide Yarns. In 18th World Textile Conference (AUTEX 2018); IOP Publishing: Istanbul, Turkey, 2018; Volume 460, p. 012053. [CrossRef]

28. Kayacan, O.; Kayacan, Ö.; Bulgun, E.; Eser, B.; Pamuk, M. Design Methodology and Performance Studies of a flexible Electrotextile Surface. Autex Res. J. 2015, 15, 153-157. [CrossRef]

29. Kazani, I.; Hertleer, C.; Mey, G.D.; Schwarz, A.; Guxho, G.; van Langenhove, L. Electrical Conductive Textiles Obtained by Screen Printing. Fibers Text. East. Eur. 2012, 1, 57-63.

30. Kim, H.; Lee, S. Evaluation of Laundering Durability of Electro-conductive Textile Dip-coated on Para Aramid Knit with Graphene/Waterborne Polyurethane Composite. Fibers Polym. 2018, 19, 2351-2358. [CrossRef]

31. Kivanc, U.; Bahadir, S.K. Effects of Home Laundering on Electrical Resistance of Signal Transmission Lines on Colored E-Textiles. J. Energy Power Eng. 2017, 11, 336-344. [CrossRef]

32. Komolafe, A. Reliability and Interconnections for Printed Circuits on Fabric. Ph.D. Thesis, University of Southhampton, Southhampton, UK, 2016.

33. Kye, M.J.; Cho, J.; Yu, J.C.; Chang, Y.W.; Han, J.; Lee, E.; Lim, H.S.; Lim, J.A. “Drop-on-textile” patternable aqueous PEDOT composite ink providing highly stretchable and wash-resistant electrodes for electronic textiles. Dye. Pigment. 2018. [CrossRef]

34. Lee, J.C.; Lo, C.; Chen, C.C.; Liu, W. Laundering Reliability of Electrically Conductive Fabrics for E-Textile Applications. In Proceedings of the 2019 IEEE 69th Electronic Components and Technology Conference (ECTC), Las Vegas, NV, USA, 28-31 May 2019; pp. 1826-1832. [CrossRef]

35. Li, Q.; Tao, X.M. Three-dimensionally deformable, highly stretchable, permeable, durable and washable fabric circuit boards. Proc. R. Soc. A 2014, 470, 20140472. [CrossRef] [PubMed]

36. Liu, X.; Guo, R.; Shi, Y.; Deng, L.; Li, Y. Durable, Washable, and Flexible Conductive PET Fabrics Designed by Fiber Interfacial Molecular Engineering. Macromol. Mater. Eng. 2016, 301, 1383-1389. [CrossRef]

37. Linz, T. Analysis of Failure Mechanisms of Machine Embroidered Electrical Contacts and Solutions for Improved Reliability. Ph.D. Thesis, Universiteit Gent, Gent, Belgium, 2012.

38. Malm, V.; Seoane, F.; Nierstrasz, V. Characterisation of Electrical and Stiffness Properties of Conductive Textile Coatings with Metal Flake-Shaped Fillers. Materials 2019, 12, 3537. [CrossRef] [PubMed]

39. Molla, M.T.I.; Compton, C.; Dunne, L.E. Launderability of Surface-Insulated Cut and Sew E-Textiles. In ISWC '18: Proceedings of the 2018 ACM International Symposium on Wearable Computers; Association for Computing Machinery: New York, NY, USA, 2018; pp. 104-111. [CrossRef]

40. Parkova, I.; Vilumsone, A. Insulation of flexible light emitting display for smart clothing. WIT Trans. Built Environ. 2014, 137. [CrossRef]

41. Paul, G.; Torah, R.; Yang, K.; Beeby, S.; Tudor, J. An investigation into the durability of screen-printed conductive tracks on textiles. Meas. Sci. Technol. 2014, 25, 025006. [CrossRef]

42. Rotzler, S. Einfluss der Sinnerschen Faktoren Sowie der Textilen Substrate auf die Waschbarkeit Textilintegrierter Leiterbahnen. Master's Thesis, Hochschule für Technik und Wirtschaft HTW Berlin, Berlin, Germany, 2018.

43. Schwarz, A. Electro-Conductive Yarns: Their Development, Characterization and Applications. Ph.D. Thesis, Universiteit Gent, Gent, Belgium, 2011.

44. Shahariar, H.; Kim, I.; Soewardiman, H.; Jur, J.S. Inkjet Printing of Reactive Silver Ink on Textiles. ACS Appl. Mater. Interfaces 2019, 11, 6208-6216. [CrossRef]

45. Shahariar, H.; Kim, I.; Bhakta, R.; Jur, J.S. Direct-write Printing Process of Conductive Paste on Fiber Bulks for Wearable Textile Heaters. Smart Mater. Struct. 2020. [CrossRef]

46. Tadesse, M.G.; Loghin, C.; Chen, Y.; Wang, L.; Catalin, D.; Nierstrasz, V. Effect of liquid immersion of PEDOT: PSS-coated polyester fabric on surface resistance and wettability. Smart Mater. Struct. 2017, 26, 065016. [CrossRef]

47. Tadesse, M.G.; Mengistie, D.A.; Chen, Y.; Wang, L.; Loghin, C.; Nierstrasz, V. Electrically conductive highly elastic polyamide/lycra fabric treated with PEDOT:PSS and polyurethane. J. Mater. Sci. 2019, 54, 9591-9602. [CrossRef]

48. Tang, Z.; Yao, D.; Du, D.; Ouyang, J. Highly machine-washable e-textiles with highstrain sensitivity and high thermal conduction. J. Mater. Chem. C 2020, 8, 2741-2748. [CrossRef]

49. Tao, X.; Koncar, V.; Huang, T.H.; Shen, C.L.; Ko, Y.C.; Jou, G.T. How to Make Reliable, Washable, and Wearable Textronic Devices. Sensors 2017, 17, 673. [CrossRef] [PubMed] 
50. Uz Zaman, S.; Tao, X.; Cochrane, C.; Koncar, V. Market readiness of smart textile structures-Reliability and washability. In Proceedings of the Aegean International Textile and Advanced Engineering Conference (AITAE2018), Mytilene, Greece, 5-7 September 2018; IOP Publishing: Bristol, UK, 2018; Volume 459, p. 012071. [CrossRef]

51. Uz Zaman, S.; Tao, X.; Cochrane, C.; Koncar, V. Launderability of Conductive Polymer Yarns Used for Connections of E-textile Modules: Mechanical Stresses. Fibers Polym. 2019, 20, 2355-2366. [CrossRef]

52. Uz Zaman, S.; Tao, X.; Cochrane, C.; Koncar, V. Understanding the Washing Damage to Textile ECG Dry Skin Electrodes, Embroidered and Fabric-Based; set up of Equivalent Laboratory Tests. Sensors 2020, 20, 1272. [CrossRef] [PubMed]

53. Xu, B.; Eike, R.J.; Cliett, A.; Ni, L.; Cloud, R.; Li, Y. Durability testing of electronic textile surface resistivity and textile antenna performance. Text. Res. J. 2019, 89, 3708-3721. [CrossRef]

54. Yokus, M.A.; Foote, R.; Jur, J.S. Printed Stretchable Interconnects for Smart Garments: Design, Fabrication, and Characterization. IEEE Sens. J. 2016, 16, 7967-7976. [CrossRef]

55. Zeagler, C.; Audy, S.; Gilliland, S.; Starner, T. Can I Wash It?: The Effect of Washing Conductive Materials Used in Making Textile Based Wearable Electronic Interfaces; Techreport; Georgia Institute of Technology: Atlanta, GA, USA, 2013.

56. Gui, H.; Tan, S.; Wang, Q.; Yu, Y.; Liu, F.; Lin, J.; Liu, J. Spraying printing of liquid metal electronics on various clothes to compose wearable functional device. Sci. China Technol. Sci. 2017, 60, 306-316. [CrossRef]

57. Komolafe, A.; Torah, R.; Wei, Y.; Nunes-Matos, H.; Li, M.; Hardy, D.A.; Dias, T.; Tudor, M.; Beeby, S. Integrating Flexible Filament Circuits for E-Textile Applications. Adv. Mater. Technol. 2019, 4, 1900176. [CrossRef]

58. Ojuroye, O.; Torah, R.; Beeby, S. Modified PDMS packaging of sensory e-textile circuit microsystems for improved robustness with washing. Microsyst. Technol. 2019. [CrossRef]

59. Satharasinghe, A.; Hughes-Riley, T.; Dias, T. Wearable and Washable Photovoltaic Fabrics. In Proceedings of the 36th European Photovoltaic Solar Energy Conference and Exhibition. EU PVSEC, Marseille, France, 9-13 September 2019; pp. 42-45. [CrossRef]

60. Tao, X.; Huang, T.H.; Shen, C.L.; Ko, Y.C.; Jou, G.T.; Koncar, V. Bluetooth Low Energy-Based Washable Wearable Activity Motion and Electrocardiogram Textronic Monitoring and Communicating System. Adv. Mater. Technol. 2018, 1700309. [CrossRef]

61. Vervust, T.; Buyle, G.; Bossuyt, F.; Vanfleteren, J. Integration of stretchable and washable electronic modules for smart textile applications. J. Text. Inst. 2012, 103, 1127-1138. [CrossRef]

62. Veske, P.; Bauwens, P.; Bossuyt, F.; Sterken, T.; Vanfleteren, J. Development and Washing Reliability Testing of a Stretchable Circuit on Knit Fabric. Appl. Sci. 2020, 10, 9057. [CrossRef]

63. Björninen, T.; Virkki, J.; Sydänheimo, L.; Ukkonen, L. Impact of Recurrent Washing on the Performance of Electro-textile UHF RFID Tags. In Proceedings of the 2014 IEEE RFID Technology and Applications Conference (RFID-TA), Tampere, Finland, 8-9 September 2014; pp. 251-255.

64. Cai, L.; Song, A.Y.; Wu, P.; Hsu, P.C.; Peng, Y.; Chen, J.; Liu, C.; Catrysse, P.B.; Liu, Y.; Yang, A.; et al. Warming up human body by nanoporous metallized polyethylene textile. Nat. Commun. 2017, 8, 496. [CrossRef]

65. Dhanawansha, K.B.; Senadeera, R.; Gunathilake, S.S.; Dassanayake, B.S. Silver nanowire-containing wearable thermogenic smart textiles with washing stability. Adv. Nano Res. 2020, 9, 123-131. [CrossRef]

66. Fu, Y.Y.; Chan, Y.L.; Yang, M.H.; Chan, Y.C.; Virkki, J.; Björninen, T.; Sydänheimo, L.; Ukkonen, L. Experimental Study on the Washing Durability of Electro-Textile UHF RFID Tags. IEEE Antennas Wirel. Propag. Lett. 2015, 14, 466-469. [CrossRef]

67. Kazani, I.; Declerq, F.; Scarpello, M.L.; Hertleer, C.; Rogier, H.; Vande Ginste, D.; Mey, G.D.; Guxho, G.; van Langenhove, L. Performance study of screen-printed textils antennas after repeated washing. Autex Res. J. 2014, 14, 47-54. [CrossRef]

68. Martinez-Estrada, M.; Moradi, B.; Fernández-Garcia, R.; Gil, I. Impact of Manufacturing Variability and Washing on Embroidery Textile Sensors. Sensors 2018, 18, 3824. [CrossRef] [PubMed]

69. Quandt, B.M.; Braun, F.; Ferrario, D.; Rossi, R.M.; Scheel-Sailer, A.; Wolf, M.; Bona, G.L.; Hufenus, R.; Scherer, L.J.; Boesel, L.F. Body-monitoring with photonic textiles: A reflective heartbeat sensor based on polymer optical fibres. Interface 2017, 14, 20170060. [CrossRef] [PubMed]

70. Virkki, J.; Björninen, T.; Kellomäki, T.; Merilampi, S.; Shafiq, I.; Ukkonen, L.; Sydänheimo, L.; Chan, Y. Reliability of washable wearable screen printed UHF RFID tags. Microelectron. Reliab. 2014, 54, 840-846. [CrossRef]

71. Wang, S.; Chong, N.L.; Virkki, J.; Björninen, T.; Sydänheimo, L.; Ukkonen, L. Towards Washable Electrotextile UHF RFID Tags: Reliability Study of Epoxy-Coated Copper Fabric Antennas. Int. J. Antenn. Propag. 2015, 2015, 424150. [CrossRef]

72. Zhao, Z.; Yan, C.; Liu, Z.; Fu, X.; Peng, L.M.; Hu, Y.; Zheng, Z. Machine-Washable Textile Triboelectric Nanogenerators for Effective Human Respiratory Monitoring through Loom Weaving of Metallic Yarns. Adv. Mater. 2016, 28, 10267-10274. [CrossRef] [PubMed] 